\title{
Long non-coding RNA metastasis associated in lung adenocarcinoma transcript 1 (MALAT1) interacts with estrogen receptor and predicted poor survival in breast cancer
}

\author{
Nai-si Huang ${ }^{1,2, *}$, Ya-yun Chi ${ }^{1,2, *}$, Jing-yan Xue ${ }^{1,2}$, Meng-ying Liu ${ }^{1,2}$, Sheng Huang ${ }^{1,2}$, \\ Miao $\mathrm{Mo}^{2,3}$, Shu-ling Zhou ${ }^{2,4}$, Jiong $\mathbf{W u}^{1,2,5}$ \\ ${ }^{1}$ Department of Breast Surgery, Fudan University Shanghai Cancer Center, China \\ ${ }^{2}$ Department of Oncology, Shanghai Medical College, Fudan University, China \\ ${ }^{3}$ Department of Clinical Statistics, Fudan University Shanghai Cancer Center, China \\ ${ }^{4}$ Department of Pathology, Fudan University Shanghai Cancer Center, China \\ ${ }^{5}$ Collaborative Innovation Center of Cancer Medicine, China \\ "These authors contributed equally to this work
}

Correspondence to: Jiong Wu, email: wujiong1122@vip.sina.com

Keywords: MALAT1, long non-coding RNA, breast cancer, estrogen receptor

Received: March 07, $2016 \quad$ Accepted: April 27, $2016 \quad$ Published: May 13, 2016

\section{ABSTRACT}

Metastasis associated in lung adenocarcinoma transcript 1 (MALAT1), a IncRNA that was first recognized as a prognostic parameter for patient survival of stage I lung cancer, is up-regulated in multiple human malignancies, including breast cancer. However, the mechanism of its function remained elusive. In the current study, by examining MALAT1 expression on mRNA level, we demonstrated that compared with MCF10A, MALAT1 expression was up-regulated in the majority of breast cancer cell lines (9/12). In 26 pairs of estrogen receptor (ER)-positive breast cancer samples, MALAT1 expression was significantly up-regulated compared with adjacent normal tissues $(P=0.012)$. Furthermore, of 204 breast cancer patients, high MALAT1 expression was associated with positive $E R(P=0.023)$ and progesterone receptor $(P R)(P=0.024)$ status. Further analysis using TCGA database revealed that ER and its target genes PGR and CCND1, were overexpressed in MALAT1 altered group compared with unaltered group, both on the mRNA and protein level. Lastly, we verified MALAT1's prognostic value in breast cancer. At the cut-off value of $75 \%$, MALAT1 was the only independent prognostic factor of recurrence-free survival (RFS) in ER-negative patients in a multivariate Cox regression model (hazard ratio [HR] $=2.83,95 \%$ confidence interval $[\mathrm{CI}] 1.02-7.83$ ). MALAT1 overexpression was also associated with poor RFS in tamoxifen treated ER-positive breast cancer patients, which might serve as a potential biomarker to predict endocrine treatment sensitivity.

\section{INTRODUCTION}

Noncoding RNA (ncRNAs) is represented by approximately $97 \%$ of transcribed RNA molecules, and only $3 \%$ of the RNAs are protein-coding messenger RNAs [1]. Long intergenic non-coding RNAs (lncRNAs), which can range from several hundred to tens of thousands of bases, are another class of newly discovered ncRNAs. LncRNAs control transcriptional alteration, implying that the difference in IncRNA profiling between normal and cancer cells is not merely a secondary effect of cancer transformation and that lncRNAs are strongly associated with cancer progression $[2,3]$.

LncRNA metastasis-associated in lung adenocarcinoma transcript 1 (MALAT1), was first recognized as a prognostic parameter for patient survival of stage I lung adenocarcinoma or squamous cell carcinoma patients in 2003 [4]. MALAT1 is also associated with several human tumor entities, including liver cancer, renal cell carcinoma, bladder carcinoma, colorectal cancer and 
cervical cancer, and it is generally regarded as a negative predictor of tumor prognosis [5, 6]. MALAT1 facilitates cell growth, migration, and invasion in malignancies [7-9]; however, the mechanism underlying these effects has remained elusive [10].

MALAT1 is also an abundantly expressed lncRNA in primary breast cancer [12]. Mutations and deletions in the human MALAT1 gene were recently discovered in luminal breast cancer $[13,14]$. Compared with normal breast tissue, MALAT1 expression was up-regulated significantly in primary breast cancer and lymph node metastasis [15]. A recent study demonstrated that, downregulation of MALAT1 mouse mammary carcinoma model resulted in slower tumor growth accompanied by significant differentiation into cystic tumors and a reduction in metastasis [16]. Nevertheless, little is known about the mechanism through which MALAT1 exerts its oncogenic activity in breast cancer, as well as its interaction with other molecules. The aim of the current study is to explore the role of MALAT1 in breast cancer oncogenesis and its potential value as a prognostic biomarker.

\section{RESULTS}

\section{MALAT1 expression profile in breast cancer cell lines}

Expression profiles for MALAT1 were examined in 12 breast cancer cell lines. As shown in Figure 1A, compared with normal breast cell line MCF10A, MALAT1 expression was up-regulated in most breast cancer cells $(9 / 12,75.0 \%)$, including MDAMB436, MDAMB468, BT549, ZR751, MCF7, T47D, SKBR3, MDAMB231HM, and BT474.

\section{MALAT1 was overexpressed in ER-positive breast cancer tissues}

Then, we detected the expression of MALAT1 in 33 pairs of breast cancer tissues and their adjacent normal tissues. The expression of MALAT1 tended to be higher in breast cancer tissues than in normal tissue but was not significantly different between the two $(P=0.075)$. Among 33 pairs of breast cancer, 26 pairs were ER positive. MALAT1 expression was significantly up-regulated compared with adjacent normal tissues $(P=0.012)$ in ERpositive breast cancers (Figure 1B).

\section{Correlations between MALAT1 expression and ER in breast cancer patients}

To identify the clinical relevance of MALAT1 expression in breast cancer, the correlation between MALAT1 expression and tumor clinical-pathological parameters was analyzed in 204 breast cancer tissues. The expression levels of MALAT1 in breast cancer were categorized as low or high expression at the cut-off value of the median. The correlation between MALAT1 expression levels and patients' clinical-pathological characteristics was summarized in Table 1. High MALAT1 expression was associated with positive ER $(P=0.023)$ and PR $(P=0.024)$ status, as well as lower tumor grades $(P=0.025)$. Nevertheless, when stratified ER status, MALAT1 expression was not correlated with tumor grade in ER-positive group $(P=0.417)$ or ER-negative group $(P=0.055)$.

Then we used the TCGA $[17,18]$ database to testify our observation and came to similar conclusions.
A



B



Figure 1: (A) MALAT1 expression profile in 13 breast cell lines. Compared with the normal breast cell line MCF10A, MALAT1 expression was up-regulated in MDAMB436, MDAMB468, BT549, ZR751, MCF7, T47D, SKBR3, MDAMB231HM, and BT474; (B) MALAT1 expression was significantly up-regulated compared with adjacent normal tissues $(P=0.012)$ in ER-positive breast cancers. 
Table 1: Relationship between MALAT1 expression and clinical-pathological characteristics of breast cancer patients

\begin{tabular}{|c|c|c|c|c|c|}
\hline \multirow{2}{*}{ Characteristics } & \multirow{2}{*}{$N$} & \multicolumn{2}{|c|}{ MALAT1 expression } & \multirow{2}{*}{$\chi^{2}$} & \multirow{2}{*}{$P$-value } \\
\hline & & Low & High & & \\
\hline \multicolumn{6}{|l|}{ Age (years) } \\
\hline$\leq 50$ & 98 & $49(48.0 \%)$ & $49(48.0 \%)$ & \multirow{2}{*}{0.000} & \multirow{2}{*}{1.000} \\
\hline$>50$ & 106 & $53(52.0 \%)$ & $53(52.0 \%)$ & & \\
\hline \multicolumn{6}{|l|}{ Menopausal status } \\
\hline Pre & 91 & $44(41.3 \%)$ & $47(46.1 \%)$ & \multirow{2}{*}{0.179} & \multirow{2}{*}{0.778} \\
\hline Post & 113 & $58(56.9 \%)$ & $55(53.9 \%)$ & & \\
\hline \multicolumn{6}{|l|}{ Tumor size $(\mathrm{cm})^{*}$} \\
\hline$\leq 2$ & 74 & $33(32.4 \%)$ & $41(40.2 \%)$ & \multirow{2}{*}{1.357} & \multirow{2}{*}{0.308} \\
\hline$>2$ & 130 & $69(67.6 \%)$ & $61(59.8 \%)$ & & \\
\hline \multicolumn{6}{|l|}{ Lymph node status } \\
\hline Negative & 109 & $56(54.9 \%)$ & $53(52.0 \%)$ & \multirow{2}{*}{0.177} & \multirow{2}{*}{0.779} \\
\hline Positive & 95 & $46(45.1 \%)$ & $49(48.0 \%)$ & & \\
\hline \multicolumn{6}{|l|}{ Tumor grade } \\
\hline I-II & 103 & $45(47.9 \%)$ & $58(65.2 \%)$ & \multirow{2}{*}{5.558} & \multirow{2}{*}{0.025} \\
\hline III & 80 & $49(52.1 \%)$ & $31(34.8 \%)$ & & \\
\hline \multicolumn{6}{|l|}{ ER status } \\
\hline Negative & 85 & $51(50.0 \%)$ & $34(33.7 \%)$ & \multirow{2}{*}{5.565} & \multirow{2}{*}{0.023} \\
\hline Positive & 118 & $51(50.0 \%)$ & $67(66.3 \%)$ & & \\
\hline \multicolumn{6}{|l|}{ PR status } \\
\hline Negative & 91 & $54(52.9 \%)$ & $37(36.3 \%)$ & \multirow{2}{*}{5.733} & \multirow{2}{*}{0.024} \\
\hline Positive & 113 & $48(47.1 \%)$ & $65(63.7 \%)$ & & \\
\hline \multicolumn{6}{|l|}{ HER-2 status } \\
\hline Negative & 106 & $57(61.3 \%)$ & $49(57.0 \%)$ & \multirow{2}{*}{0.344} & \multirow{2}{*}{0.648} \\
\hline Positive & 73 & $36(38.7 \%)$ & $37(43.0 \%)$ & & \\
\hline \multicolumn{6}{|l|}{ LVI } \\
\hline Negative & 122 & $65(66.3 \%)$ & $57(58.8 \%)$ & \multirow{2}{*}{1.191} & \multirow{2}{*}{0.302} \\
\hline Positive & 73 & $33(33.7 \%)$ & $40(41.2 \%)$ & & \\
\hline
\end{tabular}

*Only the size of invasive tumor is included.

ER: estrogen receptor; PR: progesterone receptor; HER-2: human epidermal growth factor receptor-2; LVI: lymph vascular invasion. 
MALAT1 was amplified or up-regulated in $7 \%$ of breast cancer cases (Figure 2A). ER expression was moderately correlated on mRNA level $($ Pearson $=0.38$, Spearman $=$ 0.37). Further analysis revealed that, ER was up-regulated in MALAT1 altered group compared with unaltered group, both on the mRNA level $(P<0.001)$ and protein level $(P=0.002)$. ER's target genes, $P G R$ and $C C N D 1$ were also overexpressed in MALAT1 altered group, indicating MALAT1 might play a role the regulation of ER expression in breast cancer (Figure 2B-2E).

\section{The prognostic role of MALAT1 in breast cancer}

In 204 breast cancer patients, the median follow-up time was 65.0 months (IQR: 47.0-72.2). 41 cases (20.9\%) developed recurrence, and 22 patients (11.2\%) died (18 patients died of breast cancer, three patients died of other malignant tumors, and one patient died of heart disease). At the cut-off value of $75 \%$ of MALAT1's expression, in the ER-positive group, the 5-year RFS rates of the low expression group versus the high expression group were $76.3 \%$ and $76.1 \%$, respectively $(P=0.696$; Figure $3 \mathrm{~A})$.
In the ER-negative group, the 5-year RFS rates were $81.4 \%$ and $63.7 \%$, respectively ( $P=0.076$; Figure $3 \mathrm{~B})$. No difference was detected in OS. MALAT1 expression level, tumor size, lymph node status, and lymphovascular invasion (LVI) status were included in a multivariate Cox regression analysis. As shown in Table 2, MALAT1 expression level was the only independent prognostic factor of poor RFS in the ER-negative patients; however, it did not have prognostic value in the ER-positive subset of patients.

Next, we limit the cohort to 57 tamoxifen treated ER-positive breast cancer patients and re-analyzed our data. Although no statistically significance was detected due to small sample size and few recurrences, the RFS rates for MALAT1 high expression versus low expression group were $78.6 \%$ and $83.7 \%(P=0.576)$, respectively (Figure 4A). Then we used Gyorffy's dataset [19] to conduct survival analysis and found that, in 161 tamoxifen treated ER-positive patients, high MALAT1 expression level predicted poor RFS (HR $=2.56,95 \%$ CI: $1.04-6$, $P=0.034)$ at the cut-off value of $75 \%$, which was consistent with the result of our cohort (Figure 4B).

A Altered in $80(7 \%)$ of 1105 cases/patients

MALAT1

Genetic Alteration



B







D






Figure 2: MALAT1 correlated with ER and its downstream genes' expression. (A) MALAT1 was amplified or up-regulated in 7\% of breast cancer cases; (B) MALAT1 and ER expression was moderately correlated on the mRNA level; (C) MALAT1 was related to ER expression on the mRNA and protein level; (D) and (E), MALAT1 was related to the expression of ER's target genes, PGR and CCND1, on the mRNA and protein level. 


\section{DISCUSSION}

Accumulating evidence has shown that lncRNAs play a critical biological role in cellular development and human diseases [20-22]. MALAT1, also known as nuclear-enriched abundant transcript 2 (NEAT2), is highly expressed in mammalian species [23]. Many of MALAT1's vital functions are related to nuclear processes, such as gene splicing, gene expression and nuclear organization [14]. In multiple human cancers, MALAT1 plays a role in carcinogenesis, including tumor cell proliferation, apoptosis, migration and metastasis [24-26]. In the present study, our results indicated that MALAT1 expression was up-regulated in multiple breast cancer cell lines compared with normal breast cell line, as well as in ER-positive breast cancer tissues compared with adjacent normal breast cancer tissues. These data correlated with previous studies indicating that MALAT1 was overexpressed in primary breast cancer and promoted proliferation of breast cancer cells [27-29]. We identified for the first time that MALAT1 expression was correlated with ER and its target genes in breast cancer, providing a new perspective in MALAT1 function. The mechanism through which MALAT1 regulates ER expression remained to be explored. Arun et al. reported in their study that ESR1, coding for ER, was found to be alternatively spliced in its 5' untranslated region (UTR) in MALAT1 knock-down samples; however, it remains to be further investigated whether the alternative splicing of ESR1 confers the stability of ER [16].

A

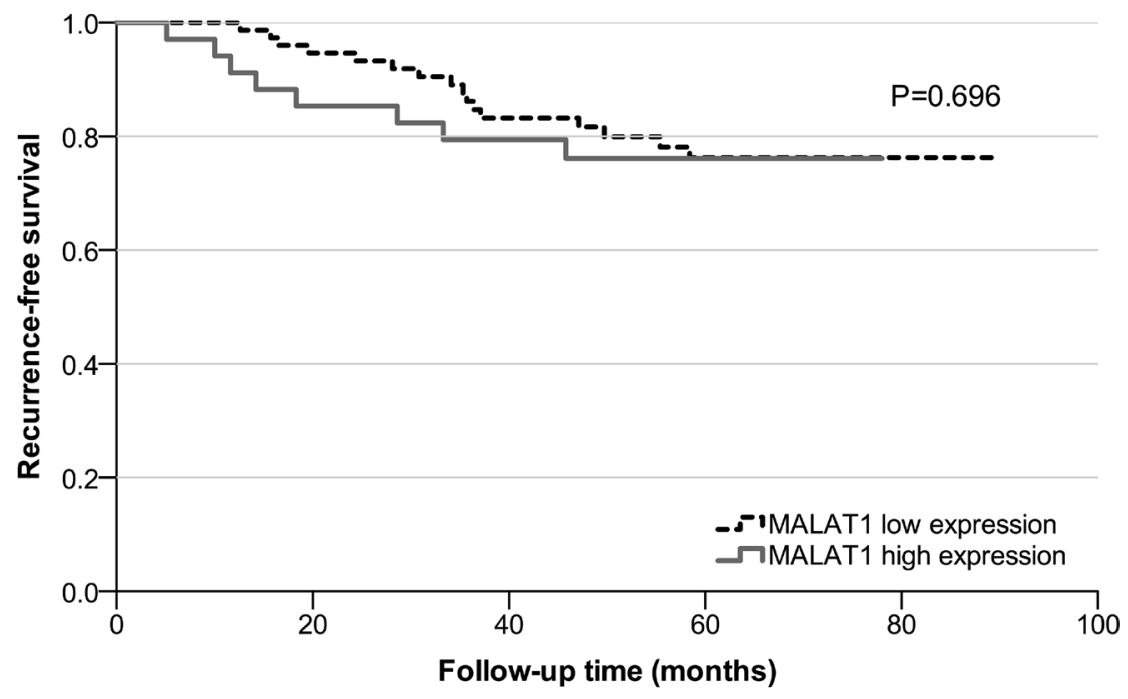

B



Figure 3: Survival analysis in breast cancer patients based on MALAT1 expression. (A) Recurrence-free survival based on low MALAT1 expression versus high MALAT1 expression in the ER-positive group; (B) Recurrence-free survival based on low MALAT1 expression versus high MALAT1 expression in the ER-negative group. 
Table 2: Multivariate Cox regression analysis of prognostic factors for RFS in breast cancer patients according to ER status

\begin{tabular}{lcccccc}
\hline \multirow{2}{*}{\multicolumn{1}{c}{ Variables }} & \multicolumn{3}{c}{ ER-positive } & \multicolumn{3}{c}{ ER-negative } \\
\cline { 2 - 7 } & HR & $\mathbf{9 5 \%}$ CI & $\boldsymbol{P}$-value & HR & $\mathbf{9 5 \%}$ CI & $\boldsymbol{P}$-value \\
\hline MALAT-1 expression high vs. low & 1.54 & $0.62-3.80$ & 0.355 & 2.83 & $1.02-7.83$ & $\mathbf{0 . 0 4 5}$ \\
Lymph node status positive vs. negative & 4.43 & $1.15-17.05$ & $\mathbf{0 . 0 3 0}$ & 0.98 & $0.29-3.33$ & 0.973 \\
Tumor size $\leq 2 \mathrm{~cm}$ vs. $>$ 2 cm & 4.18 & $0.94-18.54$ & $\mathbf{0 . 0 6 0}$ & 2.23 & $0.61-6.75$ & 0.250 \\
LVI positive vs. negative & 0.87 & $0.32-2.35$ & 0.785 & 2.99 & $0.91-9.80$ & 0.071 \\
\hline
\end{tabular}

ER: estrogen receptor; CI: confidential interval; HR: hazard ratio; LVI: lymph vascular invasion.

The prognostic value of MALAT1 in breast cancer was controversial in our study. High MALAT1 expression was an independent predictor of poor RFS in ER-negative patients, while not in ER-positive patients. Possible reasons include patients receiving various endocrine therapies that potentially affected MALAT1 expression and its predictive role; and relative short follow-up time. Several signaling pathways have been proposed to explain the oncogenesis of MALAT1 in breast cancer. Jin et al. demonstrated a reciprocal negative control relationship between MALAT1 and miR-1; MALAT1 might exert its function through the $\mathrm{miR}-1 / \mathrm{slug}$ axis [27]. Bamodu et al. identified MALAT1 as a mediator of KDM5B oncogenic potential in triple-negative breast cancer, and could be reversed by hsa-miR-448 [30]. Furthermore, MALAT1 reversed the inhibitory effect of miR-124 on breast cancer proliferation and was involved in the cyclin-dependent kinase 4 (CDK4) expression [29].
Most interestingly, we uncovered that high MALAT1 expression level was associated with tamoxifen treatment failure in ER-positive breast cancer. As MALAT1 might play a role in regulating ER expression [16], it is possible that MALAT1 affects tamoxifen resistance in an ER-dependent manner. Previous studies also demonstrated relevant variation in MALAT1 transcript abundance in tamoxifen-treated and untreated ER-positive breast cancer $[12,31,32]$. Taken together, MALAT1 might induce tamoxifen resistance via regulating transcription and splicing of ESR 1 and affecting ER signaling. MALAT1 may provide a new perspective in tamoxifen resistance mechanism and help to develop new treatment target for ER-positive breast cancer. Meanwhile, MALAT1 could serve as a potential biomarker for endocrine therapy sensitivity, which is essential for personalized treatment in these patients.
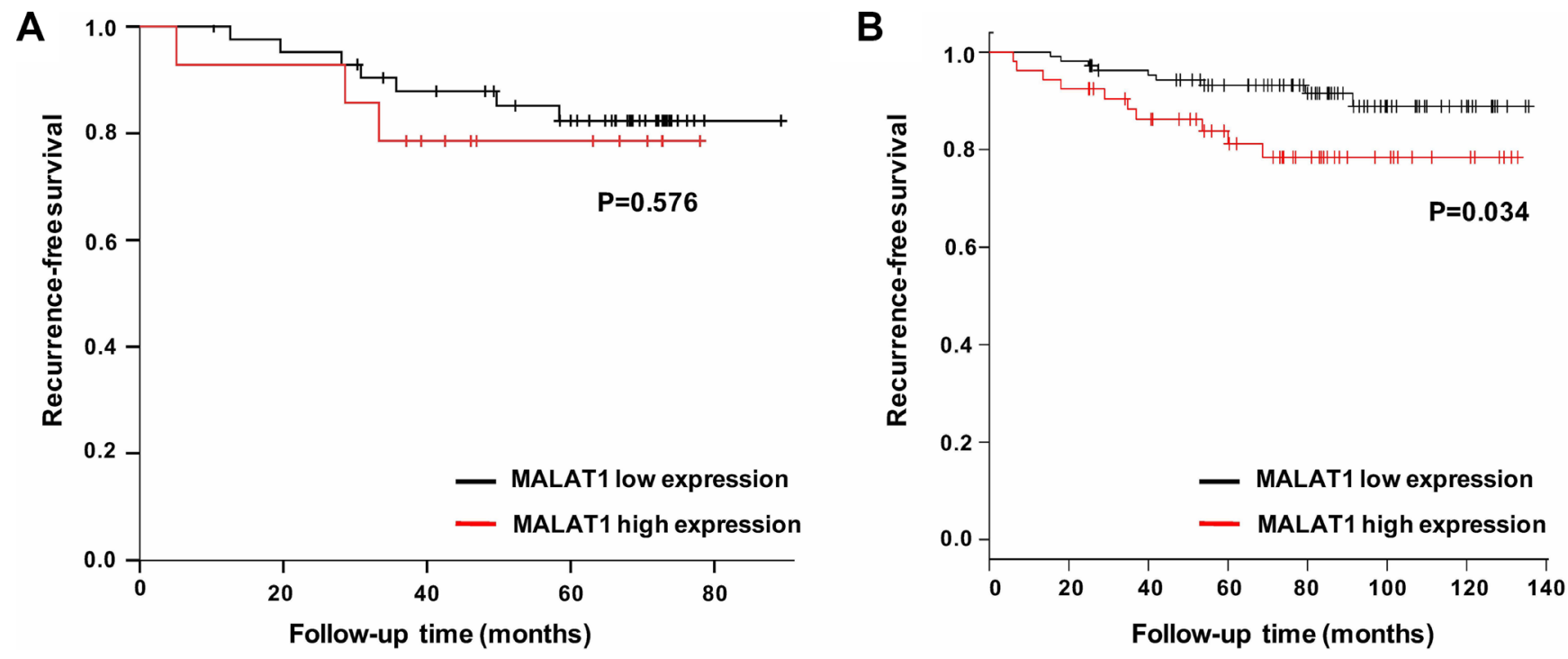

Figure 4: Survival analysis in tamoxifen treated ER-positive breast cancer patients based on MALAT1 expression. (A) Recurrence-free survival based on low MALAT1 expression versus high MALAT1 expression in a cohort of 57 patients in our institute; (B) Recurrence-free survival based on low MALAT1 expression versus high MALAT1 expression in Gyorffy's dataset of 161 patients. 


\section{MATERIALS AND METHODS}

\section{Cell line and regents}

Twelve breast cell lines and one normal breast cell line (MCF10A) were obtained from the cell bank of our lab. ZR751, ZR7530, MCF7, SKBR3, BT474 and T47D cells were cultured in RMPI1640 medium. MDAMB231 and MDAMB231HM cells were cultured in F15 medium. MDAMB436, MDAMB468, and BT549 were cultured in DMEM medium. MCF10A was cultured in F12/DMEM 1:1 medium. All cells were cultured with $10 \%$ fetal bovine serum (FBS), 100 units $/ \mathrm{ml}$ penicillin, and $100 \mathrm{ug} / \mathrm{ml}$ streptomycin at $37^{\circ} \mathrm{C}$ and $5 \% \mathrm{CO}_{2}$. The pathological features of 13 cell lines are listed in Supplementary Table 1.

\section{Patient samples}

Our study included two cohorts of patients. Firstly, 33 pairs of primary non-metastatic breast tumors and their matched adjacent normal tissues were obtained. Next, breast cancer tissues of 204 patients treated in our clinic between 2007 and 2009 were included. The following exclusion criteria were applied: (1) metastatic breast cancer, (2) patients who received neo-adjuvant chemotherapy prior to surgery and (3) patients who were diagnosed with recurrent breast cancer upon surgery. All breast samples were obtained and frozen in liquid nitrogen immediately after surgery and stored in $-80^{\circ} \mathrm{C}$ freezers in the tissue bank in Fudan University Shanghai Cancer Center (FUSCC) until RNA extraction. The percentage of cancer components exceeded $90 \%$ in each breast cancer sample. Pathology diagnosis, ER, PR, and HER2 status were reviewed by academic pathologists according to the World Health Organization (WHO) classification and American Society for Clinical Oncology (ASCO) guidelines. Patient baseline characteristics and follow-up information were included in the final analysis. Informed consent was obtained from all subjects in the current study. The Ethical Committee of FUSCC for Clinical Research approved the protocol of the study.

\section{RNA extraction and quantitative RT-PCR}

Total RNA was extracted from clinical specimens and cell lines using TRIzol reagent (Invitrogen) following the manufacturer's protocol. After converting total RNA to cDNA in a reverse transcription (RT) reaction, cDNA templates were mixed with genespecific primers for MATAT-1 (forward primer AAAGCAAGGTCTCCCCACAAG, reverse primer GGTCTGTGCTAGATCAAAAGGCA) and internal control GAPDH. Quantitative real time polymerase chain reaction (qRT-PCR) was used to quantitate MALAT1 expression. qRT-PCR was performed with the ABI7900 system. Melting curve analysis was used to monitor the specificity of the PCR products. The PCR for each sample was performed in triplicate. In addition, 2-delta $\mathrm{Ct}$ values were used to determine relative expression.

\section{Statistical and bioinformatics analysis}

A paired Wilcoxon signed rank test was used to examine MALAT1 expression in breast cancer tissues versus adjuvant normal tissues. A Pearson's $\chi^{2}$ test was performed to detect the correlation between MALAT1 expression and breast cancer clinical-pathological characteristics. In survival analysis, we used a Kaplan-Meier and Cox proportional hazards model to examine whether MALAT1 expression impacted prognosis. Two-tailed $P$-values were adopted, and $P<0.05$ was considered significant. All statistical analyses were performed using SPSS version 20.0 (SPSS Inc., Chicago, IL, USA) and SAS version 9.2 (SAS Institute Inc., Cary, NC, USA). Oncoprint analysis of the provisional breast cancer TCGA dataset were conducted via http://www. cbioportal.org. In the survival analysis of Gyorffy's dataset, the cut-off value of MALAT1 was set at $75 \%$ and only Tamoxifen treated ER-positive patients were included.

\section{ACKNOWLEDGMENTS}

None.

\section{CONFLICTS OF INTEREST}

None.

\section{GRANT SUPPORT}

This work was supported by the National Natural Scientific Foundation of China (81272924, 81472456).

\section{REFERENCES}

1. Mattick JS. The genetic signatures of noncoding RNAs. PLOS Genet. 2009; 5:e1000459. doi: 10.1371/journal. pgen. 1000459.

2. Huarte M, Rinn JL. Large non-coding RNAs: missing links in cancer? Hum Mol Genet. 2010; 19:R152-161. doi: 10.1093/hmg/ddq353.

3. Tsai MC, Spitale RC, Chang HY. Long intergenic noncoding RNAs: new links in cancer progression. Cancer Res. 2011; 71:3-7. doi: 10.1158/0008-5472.

4. Ji P, Diederichs S, Wang W, Böing S, Metzger R, Schneider PM, Tidow N, Brandt B, Buerger H, Bulk E, Thomas M, Berdel WE, Serve $\mathrm{H}$, et al. MALAT-1, a novel noncoding RNA, and thymosin beta4 predict metastasis and survival in early-stage non-small cell lung cancer. Oncogene. 2003; 22:8031-41.

5. Shen L, Chen L, Wang Y, Jiang X, Xia H, Zhuang Z. Long noncoding RNA MALAT1 promotes brain metastasis by inducing epithelial-mesenchymal transition in lung cancer. 
J Neuro-Oncol. 2015; 121:101-8. doi: 10.1007/s11060-0141613-0.

6. Schmidt LH, Spieker T, Koschmieder S, Humberg J, Jungen D, Bulk E, Hascher A, Wittmer D, Marra A, Hillejan L, Wiebe K, Berdel WE, Wiewrodt R, et al. The long noncoding MALAT-1 RNA indicates a poor prognosis in non-small cell lung cancer and induces migration and tumor growth. J Thorac Oncol. 2011; 6:1984-92. doi: 10.1097/ JTO.0b013e3182307eac.

7. Jiao F, Hu H, Yuan C, Wang L, Jiang W, Jin Z, Guo Z, Wang L. Elevated expression level of long noncoding RNA MALAT-1 facilitates cell growth, migration and invasion in pancreatic cancer. Oncol Rep. 2014; 32:2485-92. doi: 10.3892/or.2014.3518.

8. Okugawa Y, Toiyama Y, Hur K, Toden S, Saigusa S, Tanaka K, Inoue Y, Mohri Y, Kusunoki M, Boland CR, Goel A. Metastasis-associated long non-coding RNA drives gastric cancer development and promotes peritoneal metastasis. Carcinogenesis. 2014; 35:2731-9. doi: 10.1093/carcin/ bgu200.

9. Han Y, Liu Y, Nie L, Gui Y, Cai Z. Inducing cell proliferation inhibition, apoptosis, and motility reduction by silencing long noncoding ribonucleic acid metastasis-associated lung adenocarcinoma transcript 1 in urothelial carcinoma of the bladder. Urology. 2013; 81:209.e1-7. doi: 10.1016/j. urology.2012.08.044.

10. Gutschner T, Hämmerle M, Diederichs S. MALAT1 - a paradigm for long noncoding RNA function in cancer. J Mol Med. 2013; 91:791-801. doi: 10.1007/s00109-013-1028-y.

12. Guffanti A, Iacono M, Pelucchi P, Kim N, Soldà G, Croft LJ, Taft RJ, Rizzi E, Askarian-Amiri M, Bonnal RJ, Callari M, Mignone F, Pesole G, et al. A transcriptional sketch of a primary human breast cancer by 454 deep sequencing. BMC Genomics. 2009; 10:163. doi: 10.1186/1471-2164-10-163.

13. Ellis MJ, Ding L, Shen D, Luo J, Suman VJ, Wallis JW, Van Tine BA, Hoog J, Goiffon RJ, Goldstein TC, Ng S, Lin L, Crowder R, et al. Whole-genome analysis informs breast cancer response to aromatase inhibition. Nature. 2012; 486:353-60. doi: 10.1038/nature11143.

14. Tripathi V, Ellis JD, Shen Z, Song DY, Pan Q, Watt AT, Freier SM, Bennett CF, Sharma A, Bubulya PA, Blencowe BJ, Prasanth SG, Prasanth KV. The nuclear-retained noncoding RNA MALAT1 regulates alternative splicing by modulating SR splicing factor phosphorylation. Mol Cell. 2010; 39: 925-38. doi: 10.1016/j.molcel.2010.08.011.

15. Ga H, Chakraborty G, Lee-Lim AP, Mavrakis KJ, Wendel HG, Giancotti FG. Forward genetic screens in mice uncover mediators and suppressors of metastatic reactivation. Proc Natl Acad Sci U S A. 2014; 111:16532-7. doi: 10.1073/ pnas. 1403234111.

16. Arun G, Diermeier S, Akerman M, Chang KC, Wilkinson JE, Hearn S, Kim Y, MacLeod AR, Krainer AR, Norton L, Brogi E, Egeblad M, Spector DL. Differentiation of mammary tumors and reduction in metastasis upon Malat1 lncRNA loss. Genes Dev. 2016; 30:34-51. doi: 10.1101/gad.270959.115.
17. Gao J,Aksoy BA, DogrusozU, Dresdner G, Gross B, Sumer SO, Sun Y, Jacobsen A, Sinha R, Larsson E, Cerami E, Sander C, Schultz N. Integrative analysis of complex cancer genomics and clinical profiles using the cBioPortal. Sci Signal. 2013; 6:1. doi: 10.1126/scisignal.2004088.

18. Cerami E, Gao J, Dogrusoz U, Gross BE, Sumer SO, Aksoy BA, Jacobsen A, Byrne CJ, Heuer ML, Larsson E, Antipin Y, Reva B, Goldberg AP, et al. The cBio cancer genomics portal: an open platform for exploring multidimensional cancer genomics data. Cancer Discov. 2012; 2:401-4. doi: 10.1158/2159-8290.CD-12-0095.

19. Gyorffy, B, Lanczky A, Eklund AC, Denkert C, Budczies J, Li Q, Szallasi Z. An online survival analysis tool to rapidly assess the effect of 22,277 genes on breast cancer prognosis using microarray data of 1,809 patients. Breast Cancer Res Treat. 2010; 123:725-31. doi: 10.1007/s10549-009-0674-9.

20. Wang KC, Chang HY. Molecular mechanisms of long noncoding RNAs. Mol Cell. 2011; 43:904-14. doi: 10.1016/j. molcel.2011.08.018.

21. Guttman M, Rinn JL. Modular regulatory principles of large non-coding RNAs. Nature. 2012; 482:339-46. doi: 10.1038/ nature 10887 .

22. Zong X, Tripathi V, Prasanth KV. RNA splicing control: yet another gene regulatory role for long nuclear noncoding RNAs. RNA Biol. 2011; 8:968-77. doi: 10.4161/rna.8.6.17606.

23. Hutchinson JN, Ensminger AW, Clemson CM, Lynch CR, Lawrence JB, Chess A. A screen for nuclear transcripts identifies two linked noncoding RNAs associated with SC35 splicing domains. BMC Genomics. 2007; 8:39.

24. Praz V, Jagannathan V, Bucher P. CleanEx: a database of heterogeneous gene expression data based on a consistent gene nomenclature. Nucleic Acids Res. 2004; 32:D542-7.

25. Xu C, Yang M, Tian J, Wang X, Li Z. MALAT-1: a long non-coding RNA and its important 3' end functional motif in colorectal cancer metastasis. Int J Oncol. 2011; 39:169-75. doi: 10.3892/ijo.2011.1007.

26. Fellenberg J, Bernd L, Delling G, Witte D, ZahltenHinguranage A. Prognostic significance of drug regulated genes in high grade osteosarcoma. Mod Pathol. 2007; 20:1085-94.

27. Jin C, Yan B, Lu Q, Lin Y, Ma L. Reciprocal regulation of Hsa-miR-1 and long noncoding RNA MALAT1 promotes triple-negative breast cancer development. Tumour Biol. 2015. Epub ahead of print.

28. Chou J, Wang B, Zheng T, Li X, Zheng L, Hu J, Zhang Y, Xing Y, Xi T. MALAT1 induced migration and invasion of human breast cancer cells by competitively binding miR-1 with cdc42. Biochem Biophys Res Commun. 2016. Epub ahead of print. doi: 10.1016/j.bbrc.2016.02.102.

29. Feng T, Shao F, Wu Q, Zhang X, Xu D, Qian K, Xie Y, Wang S, Xu N, Wang Y, Qi C. miR-124 downregulation leads to breast cancer progression via LncRNA-MALAT1 regulation and CDK4/E2F1 signal activation. Oncotarget. 2016. Epub ahead of print. doi: 10.18632/oncotarget. 7578 . 
30. Bamodu OA, Huang WC, Lee WH, Wu A, Wang LS, Hsiao M, Yeh CT, Chao TY. Aberrant KDM5B expression promotes aggressive breast cancer through MALAT1 overexpression and downregulation of hsa-miR-448. BMC cancer. 2016; 16:160. doi: 10.1186/s12885-016-2108-5.

31. Loi S, Haibe-Kains B, Desmedt C, Wirapati P, Lallemand F, Tutt AM, Gillet C, Ellis P, Ryder K, Reid JF, Daidone MG, Pierotti MA, Berns EM, et al. Predicting prognosis using molecular profiling in estrogen receptor-positive breast cancer treated with tamoxifen. BMC Genomics. 2008; 9:239. doi: 10.1186/1471-2164-9-239.

32. Loi S, Haibe-Kains B, Desmedt C, Lallemand F, Tutt AM, Gillet C, Ellis P, Harris A, Bergh J, Foekens JA, Klijn JG, Larsimont D, Buyse M, et al. Definition of clinically distinct molecular subtypes in estrogen receptor-positive breast carcinomas through genomic grade. J Clin Oncol. 2007; 25:1239-46. 